\title{
Des indicateurs d'évolution du milieu et des sols pour rendre durable l'usage des savanes d'Amérique du Sud
}

\author{
Michel Brossard ${ }^{\mathrm{a}}$, Danilo López-Hernández ${ }^{\mathrm{b}}$ \\ a Pédologue, IRD, VALPÉDO, BP 64501, 34394 Montpellier cedex 5, France \\ b Biogéochimiste du sol, Laboratorio de Estudios Ambientales, IZT, Facultad de Ciencias, Universidad Central de Venezuela, \\ Caracas 1041-A. Apdo 47058, Caracas, Venezuela
}

\begin{abstract}
La gestion des ressources naturelles renouvelables est un des thèmes récurrents de notre revue. Les auteurs de cet article, pour le traiter, nous emmènent cette fois dans des régions faites de vastes étendues bien diversifiées et récemment mises en culture : les savanes d'Amérique du Sud. Après plusieurs années de mise en valeur selon différentes formes d'agriculture intensive, à base d'engrais minéraux et de pesticides, de nombreux problèmes de préservation du sol ont surgi. En considérant les enjeux du développement durable, cette situation éclaire de manière exemplaire les problèmes de temporalités, posés par l'inscription du temps court des actions ordinaires dans le temps long de l'intergénérationnel et du déploiement des processus biophysiques majeurs. Doit-on alors considérer les sols comme des ressources naturelles renouvelables? D'une grande quantité de travaux épars, il fallait faire une synthèse et interpeller la communauté scientifique sur les conséquences d'une intensification excessive. C'est ce qu'ont fait les auteurs.
\end{abstract}

La Rédaction

\section{Mots-clés : \\ Cerrado ; \\ Llanos ; \\ savanes ; \\ utilisation des terres ; \\ biogéochimie des sols; \\ Brésil ; \\ Colombie ; \\ Venezuela}

\section{Keywords:}

savanna;

Cerrado;

Llanos;

savanna;

land use;

soil biogeochemical

cycle;

Brazil;

Colombia;

Venezuela
Résumé - Jusqu'au milieu du siècle passé, les savanes d'Amérique du Sud ont été relativement peu concernées par l'activité agricole et sylvicole, étant un lieu d'élevage bovin extensif. Depuis, tant dans le Cerrado au Brésil que dans les Llanos de Colombie et du Venezuela, pâturages cultivés, céréales et légumineuses à grains se sont imposés sur de grandes surfaces. L'occupation des terres est également sylvicole avec l'introduction d'espèces exogènes. Ce développement de nouvelles activités a été possible avec des schémas de fertilisation inorganique importants. Après une revue comparative du mécanisme de colonisation agricole des Llanos et du Cerrado et de l'examen de quelques caractéristiques biogéochimiques des sols, nous montrons l'importance de la relation de ces milieux avec des enjeux environnementaux actuels et la nécessité de produire de nouveaux indicateurs pour l'usage des savanes.

\begin{abstract}
Soil and environmental sustainability indicators for South America's savannas. South American savannas cover some 2.69 million sq km across Brazil, Colombia, Venezuela and Bolivia. On a world-wide scale they represent one of the most extensive land areas with agricultural potential for crop and forestry production. Besides, these vast stretches of land are also the main alternative for avoiding the extension of agriculture to fragile tropical areas of higher ecological risk, such as the tropical rain forests and hillsides. Over the past four decades introduced pastures consisting chiefly of African species, in particular from the genus Brachiaria and Andropogon, and cropland have replaced the natural vegetation having low nutrient requirement in the savannas (Llanos) of Colombia and Venezuela, as well as in the Cerrado (Brazil). Intensive reforestation schemes have also taken place with alien species such as Pinus and Eucalyptus for pulpwood and timber production. The introduction of African grassland species and annual and perennial crops were made possible through the use of intensive fertilization, particularly with inorganic amendments. Following a preliminary comparative review of the colonization process by agriculture in the Llanos and Cerrado and a survey of some soil biogeochemical features, we show the crucial relation between these environments and current environmental stakes and the need to generate new indicators for the use of savanna areas.
\end{abstract}

$\overline{\text { Auteur correspondant : }}$ M. Brossard, brossard@mpl.ird.fr 


\section{Introduction}

Les savanes représentent $43 \%$ de la superficie terrestre, ce qui en fait le premier biome intertropical occupant de vastes étendues en Amérique du Sud, en Afrique, en Australie et en Asie du Sud. Leur composition végétale est caractérisée par des associations de plantes herbacées avec la présence ou non d'arbres. Le climat est contrasté, avec une alternance nette d'une saison des pluies et d'une saison sèche.

Les savanes sud-américaines, d'une superficie de 2,69 millions de $\mathrm{km}^{2}$ (Tab. 1), sont présentées comme une des plus grandes aires mondiales de terres ayant un potentiel de développement agricole et sylvicole ${ }^{1}$. Cependant, toutes les surfaces ne peuvent être dévolues à ces mises en valeur, compte tenu de contraintes physiques du milieu. De plus, les cultures récentes en savanes, comme dans d'autres régions tropicales, sont le siège de contraintes biologiques (maladies) produites par des champignons et des bactéries endémiques. De même, de nombreux invertébrés, souvent des insectes sociaux, habitants naturels de ces milieux, deviennent des prédateurs des espèces végétales récemment introduites suite à la disparition de leurs ressources trophiques habituelles.

Mais, en même temps, il faut bien admettre que les grandes extensions de savanes sont une alternative à l'expansion agricole vers des aires tropicales qui sont actuellement considérées écologiquement plus fragiles ou peu adaptées à certaines spéculations agricoles intensives, comme les versants montagneux et la forêt humide (López-Hernández, 1998).

Dans ces écosystèmes de savanes, les niveaux de nutriments offerts par les sols aux plantes, la saisonnalité pluvieuse bien marquée, en général six mois de pluies très intenses, avec de courtes petites saisons sèches dans le Cerrado, font que les contenus de matière organique (MO) des sols et les éléments qui sont associés aux processus de production-décomposition $(C, N, S, P)$ sont extrêmement variables comparés à des sols d'autres biomes (Brossard et al., 1997; Chapuis-Lardy et al., 2001). De même, l'activité biologique de la méso- et de la microfaune ainsi que des microorganismes du sol est directement liée aux niveaux des ressources trophiques et à la forte saisonnalité du climat (Araujo et López-Hernández, 1999 ; Nethuzhilin et al., 1999).

L'introduction de plantes africaines pérennes et de cultures annuelles en savanes a été uniquement possible avec des schémas rigoureux de fertilisation inorganique (N, P, K et amendements calcaires), car, à de rares exceptions près, les sols profondément altérés de

\footnotetext{
${ }^{1}$ Voir les textes et les programmes des organismes gouvernementaux concernés : EMBRAPA au Brésil, ICA (aujourd'hui CORPOICA) en Colombie, FONAIAP (aujourd'hui INIA) au Venezuela, de même que la littérature de centres internationaux comme le CIAT. Pour la signification des sigles, voir tableau 2.
}

ces régions présentent une fertilité chimique naturelle limitée et un caractère acide (Tab. 1). Les teneurs de phosphore et d'azote phytodisponibles sont généralement peu élevées (Medina, 1987; Hernández-Valencia et López-Hernández, 1999).

Il est maintenant clair que les modifications introduites par les transformations de ces paysages au Brésil (Henriques, 2003) et au Venezuela concernent de vastes territoires, et la rapide extension des infrastructures depuis 1985 conduit actuellement à éloigner de plus en plus l'activité agropastorale des centres urbains (Théry et de Mello, 2005). La transformation du biome doit être prise en compte dans son ensemble. Aussi, les leçons que nous pouvons tirer de cette récente intervention humaine dans les savanes doivent conduire à nourrir les débats et les réflexions sur le domaine intertropical (Encadré1). Notre propos est de faire une première revue comparative des mécanismes de la colonisation agricole des Llanos et du Cerrado, en nous focalisant par la suite sur la nécessité d'identifier des indicateurs caractérisant les processus biogéochimiques qui seraient affectés. Ce travail s'inscrit dans la poursuite de nos recherches sur l'analyse de l'état des sols dont la mise en culture est nouvelle, et l'observation et la modélisation des processus modifiés par les pratiques agropastorales introduites.

\section{L’agriculture en savanes}

Les caractéristiques du milieu physique ont fait considérer, pendant des décennies, le biome des savanes américaines comme inapte à la production agricole. Leur usage était réservé à l'élevage extensif et à des activités récréatives. Ce qui en soi est paradoxal. En effet, dans les savanes africaines, berceau de l'humanité, de nombreuses sociétés africaines ont réussi à adapter des pratiques agricoles, tandis que dans les savanes américaines, la présence d'indigènes depuis au moins à 12000 ans BP (Barbosa et Schmiz, 1998) n'a pas produit le même effet.

Au Venezuela, jusqu'à la première moitié du $X X^{\mathrm{e}}$ siècle, l'agriculture de savanes, essentiellement de subsistance, se limitait aux sols développés sur les dépôts récents des marges alluviales (López-Hernández et Ojeda, 1996). Jusqu'aux années 1960, les savanes sudaméricaines ont été globalement peu atteintes par l'activité anthropique. Elles étaient dévolues à un élevage extensif avec usage du feu pour éliminer le matériel lignifié des espèces natives (Tab. 1) et permettre le recrû d'herbacées tendres et plus appétantes (Kornelius et al. 1979; López-Hernández, 1995; Lopes et al., 1999; HernándezValencia et López-Hernández, 1999). À ces usages, il faut ajouter l'exploitation parallèle de la grande biodiversité de ces milieux (Barbosa et Schmiz, 1998; Oliveira et Marquis, 2002). Enfin, il convient de rappeler que la sidérurgie de l'État du Minas Gerais, au Brésil, a été l'un 


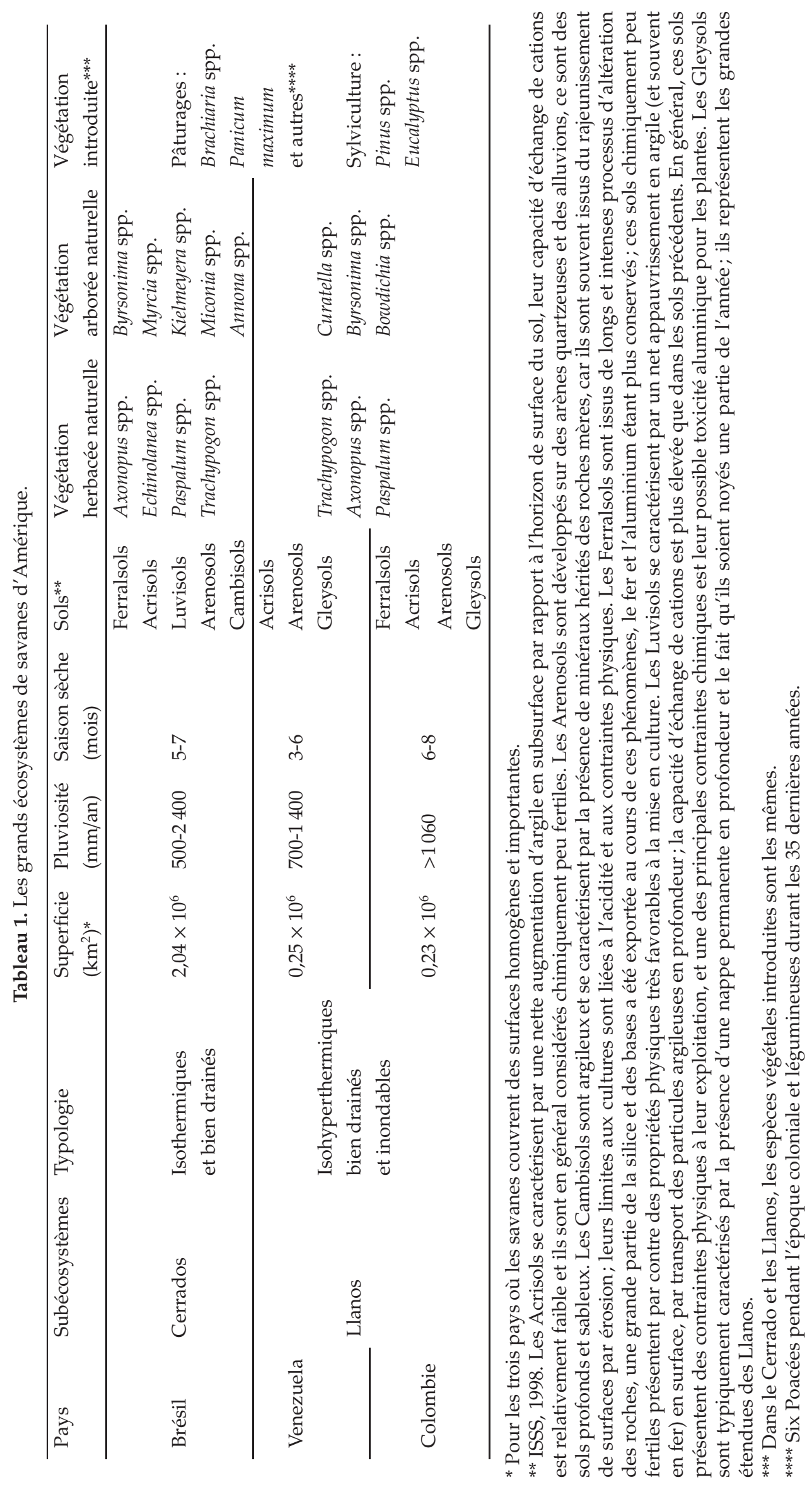




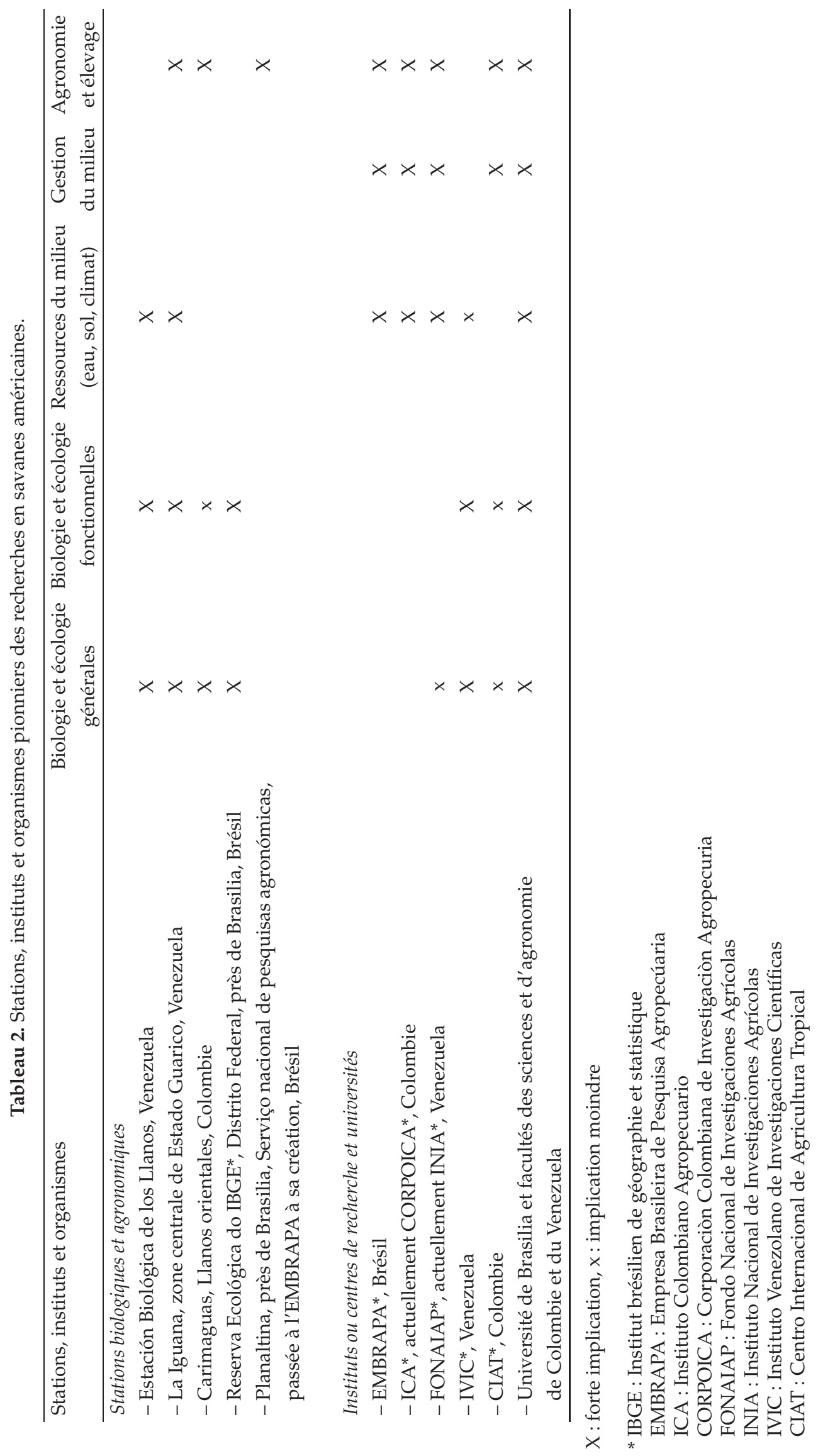


Encadré 1. Le contexte des extrapolations à d'autres milieux tropicaux

En relation aux sols. Les sols les plus cultivés des savanes américaines sont en général profonds, d'une texture relativement homogène, profondément altérés, acides à très acides; ce sont des Rhodic ou Acric Ferralsols de la classification FAO. On en rencontre de semblables dans les régions du nord du Mozambique, en Zambie, en Tanzanie et en Afrique centrale. Une partie de l'expérience acquise peut être mise en comparaison avec ce qui a été fait dans ces pays, mais aussi dans certaines régions du Zimbabwe et du Kenya.

En relation aux grandes zones agroclimatiques (sensu Pieri, 1989). Les biomes du Cerrado et des Llanos portent une végétation naturelle de type savane arbustive et arborée, plus arborée dans le Cerrado ; la pluviosité est comprise entre 700 et 1400 mm dans les Llanos, avec une saison sèche plus étendue et une amplitude pluviométrique plus importante dans le Cerrado. Ils présentent des similarités bioclimatiques avec les savanes subsahariennes qui s'étendent d'est en ouest du continent africain. Ces domaines soudano-sahélien, soudanais et soudano-guinéen portent une végétation naturelle de type arbustive et arborée, où la pluviosité est comprise entre 500 et $1100 \mathrm{~mm}$. Cette zone revêt une grande importance pour l'avenir agricole de 16 pays d'Afrique centrale.

des principaux destinataires du charbon végétal, produit artisanal de la coupe à blanc des savanes arborées et des forêts du Cerrado (Anonyme, 1993; de Carvalho et Muniz, 1996).

L'agriculture ne prend de l'importance dans les savanes de l'Orénoque colombo-vénézuéliennes que lorsque est mis en place un schéma de production lié à des apports élevés d'engrais solubles et d'intrants phytosanitaires (Sánchez, 1989; ; López-Hernández et Ojeda, 1996). Dans le Cerrado, au Brésil, le mécanisme est similaire (Goedert, 1983; Lopes et al., 1999), fruit d'une politique agricole gouvernementale très volontariste. L'extension des productions végétales s'accompagne d'une introduction ou d'une production locale de cultivars améliorés et du développement de l'irrigation qui n'était pas initialement prévue. Au cours des quarante dernières années, tant dans la zone du Cerrado que dans les Llanos, les pâturages naturels, adaptés à ces sols, ont été remplacés par des pâturages cultivés avec des espèces introduites d'Afrique (Tab. 1), principalement les genres Brachiaria et Andropogon (Pizarro et al., 1996). De même, en raison d'un contexte pédo-bio-climatique favorable, des reforestations importantes ont été mises en place avec introduction de pins et d'eucalyptus à des fins de production de bois et de pâte à papier (Tab. 1). Il n'y a pas eu de création de nouvelles variétés d'espèces arborées et fourragères depuis quinze ans, alors que l'effort a été maintenu pour les graminées et les légumineuses à grains.

\section{Les problèmes associés à l'agriculture intensive}

L'utilisation agricole intensive des savanes sudaméricaines a conduit au développement de tout un segment industriel de machinisme agricole fournissant les outils de labour, de semis, de récolte et d'épandage d'engrais et de produits phytosanitaires pour le contrôle des adventices, des maladies et des ravageurs. De plus, ces activités ont favorisé l'extension rapide des voies de circulation routières. Ce mécanisme de mise en place de techniques a été fortement soutenu, dans le cas du Brésil et du Venezuela, par des politiques volontaristes de subventions. Au Brésil, cela s'est en partie fait indirectement en mettant à disposition la terre à des prix très bas. L'opération s'y est développée à partir des années cinquante avec la décision de l'implantation de la nouvelle capitale au centre de la région du Cerrado. Au Venezuela, cela s'est produit à partir des années soixante-dix (LópezHernández et Ojeda, 1996; Lopes et al., 1999; Waniez, 1992).

Il est évident que les techniques mises en place font faire un bond spectaculaire à la production (Sá, 1999), tant des céréales et des légumineuses à grains que des pâturages. Les années passant, des détériorations des propriétés physiques des sols apparaissent (compactions, naissance des processus érosifs), ainsi que des dérèglements chimiques nutritionnels. La mécanisation intensive et continue favorise la compaction du sol (FAO, 1992; Marcano et al., 1993), par exemple via l'emploi des pulvériseurs à disques (diskplugh importé des États-Unis). Pour le Cerrado, l'altération des propriétés physiques n'est pas aisée à démontrer (Balbino et al., 2002), même si elle est couramment mise en avant (Resende et al., 1996) et bien que les formations de croûtes de battance et de semelles de labour, connues en milieux tempérés, aient rapidement fait leur apparition dans les savanes (Bravo et Florentino, 1999; Amézquita et al., 2004). Par ailleurs, les modifications d'infiltration et d'échanges gazeux, dues à une intense mécanisation, conduisent à changer les bilans hydriques du sol, mais aussi à modifier l'activité et la biomasse microbienne (Doran et al., 1998; Hernández, 1998; Hernández et López-Hernández, 2002; Hernández-Hernández et López-Hernández, 2002). Les termes ultimes de la dégradation des sols sont les mécanismes érosifs et la diminution de la productivité qui lui est souvent liée (Hernández et Domínguez, 2002; Stocking, 2003). Les connaissances acquises dans ces régions prennent en compte les facteurs physiques des sols, mais il manque des indicateurs de leur évolution en fonction du type de spéculation et des conditions pédo-climatiques. 
Les augmentations de production liées à un surcroît d'emploi d'engrais et de produits phytosanitaires ont entraîné des effets non encore complètement évalués dans les sols du Cerrado (Siqueira et Moreira, 1999). Du point de vue biologique, les pertes de diversité suite à la fragmentation puis à l'uniformisation des paysages posent aussi problème. Dans le cas des pâturages, la détérioration se traduit par la «dégradation » ou perte de production des pâturages introduits et l'invasion des plantes natives (Boddey et al., 1996).

\section{Les systèmes de travail du sol}

Le principal type de labour utilisé dans les systèmes de production de céréales et de légumineuses de ces savanes est le labour conventionnel (Sánchez, 1989; Lopes et al., 1999). Traditionnellement, il a pour objectif le contrôle des adventices, la préparation du lit de semences et un effet conditionneur des propriétés du sol (homogénéisation de l'horizon superficiel). De plus, ce traitement peut augmenter la porosité, donc l'aération, et favoriser l'intégration des débris végétaux aériens au sol (FAO, 1992).

À l'opposé se trouvent les systèmes de travail minimum du sol, tel le semis direct qui réduit la mécanisation au moment du semis et le contrôle des adventices à son expression minimale (FAO, 1992). Ces modes de gestion utilisent une couverture végétale morte ou vive d'au moins $30 \%$ de la biomasse aérienne. Créés et utilisés en Europe depuis plus de quarante ans, ces systèmes ont été très peu utilisés en Colombie et au Venezuela. Mais, dans le Cerrado, de sérieux problèmes de ruissellement et d'érosion en nappe dans les systèmes de production de céréales, soja et coton ont contraint, depuis 1980, les producteurs à changer de stratégie et à adopter les systèmes du travail minimum du sol (Séguy et al., 1996). Ces systèmes ont été activement diffusés par les migrants venant des régions subtropicales du Brésil ; on estime actuellement les surfaces ainsi cultivées à environ 2,5 millions ha dans le Cerrado. Les études menées montrent une réduction du ruissellement, une meilleure offre d'azote pour la culture commerciale et une conservation des teneurs de matière organique des sols (Scopel et al., 2005). Au Venezuela, ces systèmes ont été plus étudiés que réellement employés. Hétier (2003), qui a fait le bilan des travaux expérimentaux, souligne en particulier les incertitudes qui les ont jalonnés, ce qui explique qu'ils n'ont pas eu un réel rôle démonstratif. Alors qu'au Brésil les bénéfices économiques et édaphiques sont admis, les essais au Venezuela n'ont pas permis de conclure sur l'effet induit sur les propriétés édaphiques, bien que des résultats favorables sur l'infiltration de l'eau dans les sols et le bilan d'azote aient été observés (Jiménez, 1998; Lozano, 1998). Au total, au Venezuela et en Colombie, les surfaces concernées par le travail minimum du sol sont respectivement estimées à 170000 ha et 1000 ha (Rondón et al., à paraître).

\section{Les systèmes intégrés agriculture-élevage}

Bien qu'à l'origine les intérêts des éleveurs et des agriculteurs aient paru opposés, au cours des dernières années se sont peu à peu popularisés dans le Cerrado des schémas de production à cycles courts intégrés aux activités d'élevage (Spain et al., 1996). On tente ainsi de récupérer la productivité de pâturages en déclin et, simultanément, de rétablir des conditions édaphiques favorables aux cultures à cycles courts (Ayarza et al., 1998). Différentes stratégies sont offertes. Pour limiter le déclin des pâturages et remédier à l'état dégradé de vieux pâturages, le système agropastoral «barreirão » (Oliveira et al.,1996) offre la possibilité d'intégrer culture et élevage sur les mêmes surfaces grâce à l'implantation simultanée d'une céréale destinée à la production de grains lors de la première année et d'une graminée destinée au pâturage après récolte. Une autre stratégie consiste à transformer peu à peu les unités de production bovine en des unités mixtes agriculture/élevage avec des rotations pâturages/cultures à cycles courts (Rippstein et al., 1996; Spain et al., 1996; Ayarza et al., 1998; Balbino et Di Stefano, 1999). Lopes et al. (1999) résument les avantages de ces systèmes : augmentation de l'activité biologique, recyclage des nutriments plus efficace, meilleure utilisation de l'eau, offre alimentaire pour le bétail améliorée pendant la saison sèche et une moindre invasion par les adventices. Ces auteurs signalent que ces stratégies d'utilisation des savanes ont été rendues plus attractives avec les systèmes de semis direct, dans la mesure où ces derniers sont déjà établis, et que cela n'entraîne pas de gros investissements ${ }^{2}$. Depuis 1999, au Venezuela, est réalisée une évaluation agroécologique de ces systèmes intégrés dans les savanes centrales des Llanos (Bravo et al., 1999).

\section{L'expérience accumulée par l'étude agroécologique des savanes américaines}

Prétendre faire une synthèse exhaustive de l'information existante, dispersée et peu accessible, est une gageure. Toutefois, quelques grands traits de l'apport à la connaissance et à la gestion de ces régions par l'écologie et l'agronomie sont résumés et présentés au tableau 2 . La création d'une station au Venezuela il y a une cinquantaine d'années (Estación Biológica de los Llanos) a permis l'étude de savanes de l'Orénoque. Ces types

\footnotetext{
2 Signalons que cela a induit des interactions cultivateuréleveur, l'éleveur offrant les surfaces de pâturage servant à l'implantation de cultures pour 3 à 5 cycles, et le cultivateur installant au dernier cycle un nouveau pâturage.
} 
de stations jouent un rôle important compte tenu de leur implantation au centre de grandes régions et de leur pérennité. Ont été ensuite créées les stations de La Iguana et de Carimaguas, alors qu'au Brésil l'université de Brasilia développait ses programmes avec la Reserva Ecológica de 1'IBGE. La recherche agronomique et agroécologique est ainsi portée par les stations expérimentales des services nationaux. Ce bref sommaire ne saurait masquer l'influence qu'ont eue les universités nationales et les instituts ou centres de recherche comme le CIAT en Colombie, l'IVIC au Venezuela, les écoles d'agronomie de Piracicaba et de Campinas au Brésil, ainsi que le programme RADAMBRASIL d'inventaires des ressources naturelles, coordonné par le ministère des Mines et de l'Énergie, qui a produit la connaissance de base en phytogéographie et pédologie.

Au total, alors que les travaux de recherche dans ces savanes ont maintenant plus de cinq décennies, c'est seulement au cours des deux dernières que s'est profilé un corpus de travaux spécifiques sur l'agriculture des savanes et la recherche de mesures de l'évolution des paramètres du sol, soit de manière directe, soit de façon implicite.

\section{La confrontation entre production et protection du milieu}

La gestion des sols et des paysages agraires et forestiers est une nécessité inaliénable pour le maintien de la capacité de production d'aliments et de fibres. Ce discours apparaît désormais plus ou moins officiellement dans les pays étudiés. Actuellement, et de manière schématique, deux points de vue s'opposent sur la façon de concevoir l'agriculture de ces régions et le débat qui en résulte se fait sans la participation des citoyens. D'un côté, ceux qui défendent une agriculture hautement productiviste sont peu enclins à s'inquiéter des questions d'environnement. Leur unique credo est l'exportation du produit obtenu (la presse hebdomadaire brésilienne est friande de ce type de présentation). Ce groupe est constitué d'entreprises, mais aussi de familles fortement capitalisées et bien outillées. Elles étendent actuellement la frontière agricole vers $\mathrm{l}^{\prime}$ Amazonie sans prendre en considération le fait que les sols et le climat ne sont pas partout favorables à des cultures intensives à cycles courts. Un exemple actuel est celui des fronts d'occupation ouverts depuis six ans dans le nord du Mato Grosso au Brésil (Bertrand et al., 2005).

De l'autre côté, se trouvent les tenants d'une écologie politique (les verts et les écologistes), souvent universitaires dans ces pays, pour qui la protection des grands écosystèmes est un objectif primordial (Pro-Cer/WWF, 1995). Il est clair qu'aux confins des savanes sudaméricaines, nous retrouvons, lancé par la Conférence des Nations unies pour le développement (CNUED) de Rio en 1992, le débat global sur l'usage des ressources terrestres (Dias, 1993 ; Klink, 1996). Un des arguments avancés des plus intéressants est la limite de productivité par unité de surface atteinte par les systèmes conventionnels et qui promeuvent indirectement l'extension de la frontière agricole (Klink et al., 1993).

Ainsi, la recherche locale, en particulier agronomique et écologique, est confrontée à des défis pour trouver de nouvelles options de production définissant clairement les conditions de développement dans un contexte de respect de l'environnement. Ces options ne dépendent pas seulement d'approches techniques, mais aussi d'une capacité d'invention et d'expérimentation dans le cadre d'enjeux qui dépassent l'exploitation de ces milieux.

\section{Les enjeux de l'usage des savanes}

Au cours du passé récent, les enjeux du Brésil étaient liés à l'agriculture et à la production sylvicole; ils le restent en partie, même si les secteurs industriels et des services sont actuellement la principale base économique. $\mathrm{Au}$ Venezuela, ils étaient du domaine pétrolier et le restent. Dans ces pays, l'occupation des espaces des savanes a mobilisé des systèmes de production industrielle, la petite sidérurgie, et l'extension des réseaux d'énergie et des voies de communication. Mais les enjeux liés à la production de ces savanes ne sont plus uniquement une question de gestion des ressources renouvelables, vivantes et de l'eau. En effet, les enjeux d'expansion et de développement agricole de ces régions sont d'actualité, car ils prennent une dimension nationale, par les populations concernées, et transnationale, par le rôle de ces systèmes dans la production mondiale. Ceci est illustré dans le tableau 3, qui montre que, pour les éléments biogènes, les enjeux à diverses échelles d'analyse peuvent être clairement identifiés dans le cadre des connaissances actuelles. L'expansion agricole des savanes a deux dynamiques. La première est déterminée par les interactions entre les acteurs locaux et les choix politiques du pays; ainsi, les communautés et les associations d'agriculteurs, les autorités locales et régionales promeuvent une stratégie agricole locale. La seconde dynamique promeut ou ralentit l'expansion par des facteurs externes au pays considéré. En particulier depuis une quinzaine d'années, les effets externes au pays orientent des choix de cultures, d'élevage, d'opportunités de spéculations de production et, en conséquence, agissent indirectement sur le mode d'usage et de gestion des terres.

L'information disponible, abondante dans le domaine académique, l'est encore plus dans la « littérature grise » (MAS, 1994 et 1995). Il n'existe pas de vison synthétique des savanes américaines, comme il en existe pour l'Afrique de l'Ouest (Pieri, 1989). Les changements rapides qui se produisent dans une conjoncture mouvante 
Tableau 3. Les enjeux de l'usage des savanes américaines et des éléments majeurs biogènes à trois échelles d'analyse spatiale.

\begin{tabular}{|c|c|c|c|}
\hline & \multicolumn{3}{|c|}{ Échelles d'analyse } \\
\hline Thème & Globale & Savanes américaines & Locale à petite région \\
\hline $\begin{array}{l}\text { Expansion } \\
\text { de } \\
\text { l'agriculture } \\
\text { et de la } \\
\text { sylviculture }\end{array}$ & $\begin{array}{l}\text { Besoins de fibres et } \\
\text { d'aliments au niveau } \\
\text { planétaire } \Rightarrow \text { savanes } \\
\text { américaines = biodiversité } \\
\text { importante } \stackrel{y}{\Rightarrow} \text { gestion des } \\
\text { ressources renouvelables }\end{array}$ & $\begin{array}{l}\text { Intensification et diversification } \\
\Leftrightarrow \text { fragmentation du continuum des } \\
\text { paysages de savanes }\end{array}$ & $\begin{array}{l}\text { Réponses dépendant de pressions et } \\
\text { d'opportunités } \Leftrightarrow \text { gestion de l'eau } \\
\text { (quantité et qualité) } \Leftrightarrow \text { notions de } \\
\text { vulnérabilité }\end{array}$ \\
\hline Carbone & $\begin{array}{l}\text { Cycle global du carbone } \\
\text { Gaz à effet de serre }\end{array}$ & $\begin{array}{l}\text { Choix des systèmes cultivés } \Leftrightarrow \text { choix } \\
\text { des ressources renouvelables } \\
\Leftrightarrow \text { Variations couverture des sols } \\
\Leftrightarrow \text { biodiversité et bilan de carbone (C } \\
\text { exporté, pertes ruissellement et } \\
\text { érosion, gaz, sol et biomasse) }\end{array}$ & $\begin{array}{l}\text { Quantités et formes } \\
\Leftrightarrow \text { CEC et bases échangeables, } \\
\text { propriétés physiques, ressource } \\
\text { trophique pour organismes du sol }\end{array}$ \\
\hline Azote & $\begin{array}{l}\text { Cycle global de l'azote } \\
\text { Gaz à effet de serre } \\
\text { Dépendance des cours } \\
\text { mondiaux }\end{array}$ & $\begin{array}{l}\text { Gestion de la fertilisation } \Leftrightarrow \text { choix } \\
\text { des rotations et des systèmes } \\
\text { (importance légumineuses), dépôts } \\
\text { gazeux, bilan N (fixation, N exporté, } \\
\text { pertes ruissellement et érosion, gaz, } \\
\text { sol et biomasse) }\end{array}$ & $\begin{array}{l}\text { Quantités et formes } \\
\Leftrightarrow \text { Azote minéral du sol versus } \\
\text { engrais et rôle réel des } \\
\text { légumineuses dans l'acidification }\end{array}$ \\
\hline Phosphore & $\begin{array}{l}\text { Dépendance des cours } \\
\text { mondiaux et, de plus en } \\
\text { plus, de l'industrie } \\
\text { étrangère }\end{array}$ & $\begin{array}{l}\text { Gestion de la fertilisation } \Leftrightarrow \text { choix } \\
\text { des rotations et des systèmes, dépôts } \\
\text { atmosphériques, bilan } \mathrm{P}(\mathrm{P} \text { exporté, } \\
\text { pertes ruissellement et érosion, sol et } \\
\text { biomasse) }\end{array}$ & $\begin{array}{l}\text { Quantités et formes } \\
\text { phytodisponibles très variables }\end{array}$ \\
\hline Soufre & Cycle global du soufre & $\begin{array}{l}\text { Gestion de la fertilisation } \Leftrightarrow \text { choix } \\
\text { des rotations et des systèmes, devenir } \\
\text { des sols hydromorphes et des } \\
\text { marécages, dépôts atmosphériques, } \\
\text { bilan } \mathrm{S} \text { (S exporté, gaz, pertes } \\
\text { ruissellement et érosion, sol et } \\
\text { biomasse) }\end{array}$ & $\begin{array}{l}\text { Peu ou pas de } \mathrm{S} \text { phytodisponible } \\
\text { dans les sols de savanes }\end{array}$ \\
\hline $\begin{array}{l}\text { Calcium et } \\
\text { magnésium }\end{array}$ & $\begin{array}{l}\text { Cycle géochimique } \\
\text { sédimentaire }\end{array}$ & $\begin{array}{l}\text { Nature et éloignement des gisements } \\
\text { carbonatés et magnésiens, industries } \\
\text { de transformations locales }\end{array}$ & $\begin{array}{l}\text { Importance de Ca dans la régulation } \\
\text { du pH des sols acides, apport de Ca } \\
\text { et de } \mathrm{Mg} \text { phytodisponibles peu } \\
\text { abondant dans les sols, éléments } \\
\text { polluants associés (pollution } \\
\text { diffuse) }\end{array}$ \\
\hline Potassium & $\begin{array}{l}\text { Dépendance des cours } \\
\text { mondiaux et, de plus en } \\
\text { plus, de l'industrie } \\
\text { étrangère }\end{array}$ & Éloignement des centres industriels & $\begin{array}{l}\text { Peu ou pas de K phytodisponible } \\
\text { dans les sols de savanes }\end{array}$ \\
\hline
\end{tabular}

\footnotetext{
$\stackrel{4}{\rightarrow}$ conséquences

$\Rightarrow$ implique, relation avec

$\Leftrightarrow$ interrelation
}

en sont peut-être une explication (Bainville et al., 2005; Théry et de Mello, 2005). Face à ces changements rapides, la lecture proposée des enjeux permet d'offrir des indicateurs de la durabilité de ces savanes.

\section{Nécessité de développer des paramètres ou des indicateurs de mesure de la durabilité en savanes}

Les enjeux de production et de qualité du milieu posés à l'agriculture se traduisent, depuis quinze ans, par des contraintes de respect de la qualité des eaux et des sols (Doran et Parkin, 1996 ; Stocking, 2003). Le concept d'un système de production «durable » implique une approche régionalisée pour intégrer la variabilité des sols à des échelles pertinentes. Mais les évaluations ainsi faites seront éphémères, car les contraintes physiques et socioéconomiques de la région et du pays varient au cours du temps (Dumansky, 1993; García-Guadilla et LópezHernández, 1998). Aussi la définition de paramètres ou d'indicateurs de mesure de la "durabilité » s'avère-t-elle délicate, et ce d'autant qu'il y a nécessité d'intégrer des alternatives nouvelles, des techniques qui évoluent, de nouveaux systèmes de cultures, des faisabilités à géométrie variable selon les choix précédents, les opportunités, les problèmes de capitalisation et de financement, et ceci dans des systèmes complexes. Nous nous trouvons alors 
Tableau 4. Ensemble minimal de données physiques, chimiques et biologiques de suivi des critères de qualité du sol, d'après Doran et Parkin, 1996.

\begin{tabular}{|c|c|}
\hline Indicateur & Fonctions et processus régulés \\
\hline \multicolumn{2}{|c|}{ physiques } \\
\hline Texture & Rétention et transport d'eau et de nutriments \\
\hline Profondeur du sol & Productivité végétale et érosion \\
\hline Densité apparente et infiltration & Productivité, érosivité, lixiviation \\
\hline Capacité de rétention en eau & Disponibilité en eau, transport et érosion \\
\hline \multicolumn{2}{|c|}{ chimiques } \\
\hline $\mathrm{pH}$ & Fertilité \\
\hline Matière organique & Activité biologique et réserve de nutriments \\
\hline Conductivité électrique & Activité microbiologique \\
\hline $\mathrm{N}$, P et K extractibles & Fertilité et qualité des sols \\
\hline \multicolumn{2}{|c|}{ biologiques } \\
\hline C, N, P microbiens & Potentiel catalytique microbien \\
\hline $\mathrm{N}$ potentiellement minéralisable & Fertilité et productivité \\
\hline Respiration endogée & Pertes de C, activité microbienne \\
\hline
\end{tabular}

face à des échéances de travaux longues et coûteuses (Amézquita et al., 1999) ${ }^{3}$.

Dans le cadre de notre analyse, cette "durabilité » des systèmes dépend en partie du maintien ou des améliorations qui peuvent être faites aux fonctionnements physique et biogéochimique des sols. Le tableau 4 présente un ensemble minimal qui avait été proposé par la Société américaine de science du sol ; peu relayé localement, il n'a donc pas été l'objet d'une réelle appropriation (de Novais, 1996). Parmi ces paramètres, certains sont peu sensibles à des variations rapides, d'autres nécessitent une attention annuelle. Cette procédure permet d'intégrer la recherche de nouvelles stratégies de production. Si l'on suit Amézquita et al. (1999), deux aspects peuvent être retenus : (i) les paramètres édaphoclimatiques ; (ii) le diagnostic du profil du sol (qualité du sol). Pour le premier point, les données relevées à l'échelle des grandes régions $\left(>1 / 500000^{\mathrm{e}}\right)$ sont suffisantes pour faire des prédictions relativement pertinentes. Pour le second point, l'information sur l'impact des divers modes d'usage des sols et de gestion est locale et souvent à l'échelle de la parcelle. Il manque ici une transposition à l'échelle des petites régions, mais aussi une analyse plus globale visant à mettre en œuvre des mesures portant sur les compartiments biologiques, actuellement pas ou mal évalués. Il en est ainsi des biomasses et minéralomasses microbiennes $(C, N, P)$, du rôle et de l'importance réelle des mycorhizes, de l'écologie des microorganismes et des organismes invertébrés du sol et de la possible utilisation de ces derniers comme indicateurs, du rôle des activités

\footnotetext{
${ }^{3}$ Concernant ces derniers points, il est clair que les menaces qui pèsent depuis environ cinq ans sur les essais agronomiques de longue durée, pour des aspects uniquement financiers, constituent actuellement un problème qui doit être posé très clairement au sein des communautés scientifiques et des centres de décisions des politiques scientifiques. Ceci rejoint les problèmes de financement et de maintien des observatoires en géosciences, par exemple.
}

enzymatiques du sol et de leurs utilisations éventuelles comme indicateurs des changements d'états dans les sols (Tab. 5). Mais il en est ainsi aussi de données robustes qui pourraient être produites à l'aide de ces indicateurs. Qui peut donner actuellement une courbe d'évolution de la teneur en matière organique des sols de ces savanes en fonction des années de culture? Si l'on connaît raisonnablement à l'échelle régionale les surfaces de sols acides, connaît-on les surfaces concernées par la correction du $\mathrm{pH}$ et l'épaisseur de la couche de sol atteinte par ces changements chimiques? Ainsi, une des tâches les plus importantes à réaliser dans le futur concerne la production ou l'amélioration d'indicateurs d'évolution de durabilité des systèmes de production de ces savanes. Concernant les éléments biogènes, il nous paraît important d'intégrer des critères qui sont nouveaux pour ces savanes. Ils concernent aussi les eaux de surface (Tab. 5), et en particulier $\mathrm{N}$ et $\mathrm{P}$ totaux, $\mathrm{pH}$, résidus agrochimiques dans les eaux et les sédiments récents, utilisation réelle des eaux de surface en agriculture, volume et commerce de matériel d'irrigation.

Il faut cependant intégrer certains facteurs socioéconomiques, afin de lier les thèmes qui nous intéressent aux services offerts. Ainsi, par exemple, les éléments fertilisants sont de plus en plus importés dans ces régions et l'on doit se poser la question de savoir quels vont être le rôle et les attitudes, compte tenu de leurs besoins, des communautés locales et des autorités régionales face à des politiques étatiques et aux opportunités qu'elles créent. Cet exemple n'est pas anodin, le Brésil pourra devenir le principal producteur agricole au monde; mais, depuis 1998, il a perdu son indépendance de production d'engrais $^{4}$ et le producteur ne peut plus faire jouer la concurrence dans ce domaine. . . Le déclin de la fertilité

\footnotetext{
${ }^{4}$ La part de l'importation d'engrais dans la consommation était de $48 \%$ en 1990, elle est passée à $77 \%$ en 2003 (http://www.agricultura.gov.br, rubrique statistiques).
} 


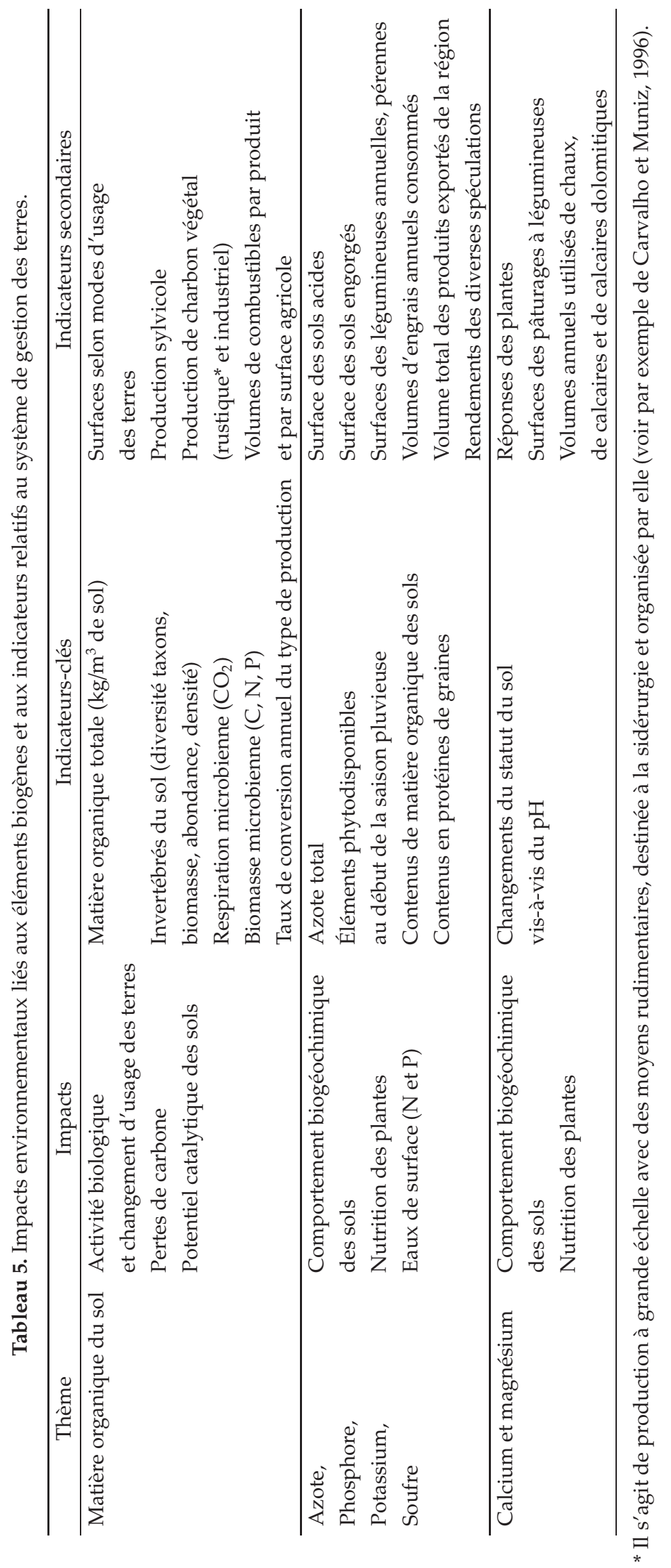


des sols n'est pas uniquement un problème technique de réduction de l'offre de nutriments du sol pour les plantes. Il interagit sur des pas de temps variables, avec tout un panel de contraintes biologiques et socioéconomiques intervenant dans le système de gestion des terres (Tab. 3).

\section{Conclusion}

Le modèle d'agriculture et de gestion des terres produit dans un écosystème donné est implicitement relié aux contraintes imposées par le milieu biophysique et les demandes socioéconomiques, particulièrement démographiques, des communautés qui sont concernées. Ainsi, chaque société produit, à un moment donné de son histoire, des systèmes en équilibre avec ces contraintes. Il y a une trentaine d'années, Van Wambeke (1971) se faisait le porte-parole des agronomes brésiliens qui se demandaient s'il fallait " convertir les savanes en terres de cultures ou les aménager en pâturages améliorés ". Les deux objectifs ont été conduits à terme. Le modèle de développement ou d'occupation (colonisation) agricole intensif des terres des savanes d'Amérique du Sud est une aventure humaine récente datant d'une cinquantaine d'années. Cette dernière n'a été d'abord qu'une simple transposition de techniques mises au point ailleurs. Il nous semble qu'il est temps de tirer des enseignements de la connaissance ainsi produite. Toutefois, le modèle conceptuel de fonctionnement de ce type de systèmes n'est pas unique, mais doit s'articuler sur l'hétérogénéité inhérente aux connaissances disciplinaires (écologie, agronomie, pédologie, sciences politiques, sociologie, économie, géographie... ). En se projetant dans une dynamique transdisciplinaire, le montage d'un modèle universel ne serait pas le plus approprié. En effet, la diversité des milieux de savanes est grande ainsi que celle des sociétés qui s'y développent. Aussi, les projets, les valeurs et les options qui seront produits dans des conditions données ne sont pas directement transposables dans un autre contexte. La vision d'une intégration au travers de processus biogéochimiques oblige à réfléchir sur la façon de connecter entre eux des éléments de connaissance acquis, de manière séparée, dans diverses disciplines. En d'autres termes, les champs de connaissances ouverts par l'expansion récente de ces frontières agricoles ne doivent pas servir seulement à assurer l'usufruit de systèmes de production déjà en place, mais ils doivent permettre de nous interroger aussi sur les transformations de ces systèmes dans le cadre des variations bio-climatiques, sociales, présentes et futures.

L'information engendrée, tant dans le Cerrado que dans les Llanos, pour surmonter les contraintes naturelles de ces savanes peut, dans les limites de l'extrapolation, nourrir des débats pour d'autres régions tropicales. Les propositions que nous faisons et qui concernent les éléments biogènes permettent d'avoir une vision plus large et de sortir d'approches « dogmatiques » sur la fertilisation des sols qui prévalent dans les pays concernés ${ }^{5}$.

\section{Références}

Amézquita, E., Friesen, D., Sanz, J.I., 1999. Indicadores de sostenibilidad: Parámetros edafoclimáticos y diagnóstico del perfil cultural. Sistemas Agropastoriles en Sabanas Tropicales de América Latina, in Guimarães, E.P., Sanz, J.I., Rao, I.M., Amézquita, M.C., Amézquita, E. (Eds), Publicación CIAT, 313, 49-64.

Amézquita, E., Thomas, R.J., Rao, I.M., Molina, D.L., Hoyos, P., 2004. Use of deep-rooted tropical pastures to build-up an arable layer through improved soil properties of an Oxisol in the Eastern Plains (Llanos Orientales) of Colombia, Agriculture, Ecosystems \& Environment, 103, 2, 269-277.

Anonyme, 1993. O Cerrado e a siderurgia, Silvicultura, 13, 48, 6-9.

Araujo, Y., López-Hernández, D., 1999. Earthworm populations in a savanna agroforestry system of Venezuelan Amazonia, Biol. Fert. Soils, 29, 413-418.

Ayarza, M.A., Vilela, L., Barcellos, A. de O., Balbino, L.C., Brossard, M., Pasini, A., 1998. Intégration culture-élevage dans les Cerrados au Brésil : une solution pour des systèmes durables, Agriculture et développement, 18, 91-98.

Bainville, S., Affholder, F., Figuié, M., Madeira Netto, J. da S., 2005. Les transformations de l'agriculture familiale de la commune de Silvânia : une petite révolution agricole dans les cerrados brésiliens, Cahiers Agricultures, 14, 1, 103-110.

Balbino, L.C., Di Stefano, J.G., 1999. Projet PROCITROPICOS : intégration de l'agriculture et de l'élevage par les systèmes de semis direct, in Rasolo, F., Raunet, M. (Eds), Gestion agrobiologique des sols et des systèmes de culture, Actes de l'atelier international du 23 au 28 mars 1998, Antsirabe, Madagascar, [Montpellier], Cirad, 409-417.

Balbino, L.C., Brossard, M., Leprun, J.C., Bruand, A., 2002. Mise en valeur des Ferralsols de la région du Cerrado (Brésil) et évolution de leurs propriétés physiques : une étude bibliographique, Étude et gestion des sols, 9, 83-104.

Barbosa, A.S., Schmiz, P.I., 1998. Ocupação indígena do cerrado: esbiço de uma hístória, in Sano, S.M., Almeida, S.P. de (Eds), Cerrado: ambiente e flora, Planaltina, DF (Brasil), EMBRAPA, 3-43.

Bertrand, J.-P., Cadier, C., Gasquès, J.G., 2005. Le crédit : un des facteurs-clés de l'expansion de la filière soja dans le Mato Grosso, Cahiers Agricultures, 14, 1, 46-52.

Boddey, R.M., Alves, B.J.R., Urquiaga, S., 1996. Nitrogen cycling and sustainability of improved pastures in the Brazilian Cerrados, in Pereira, R.C., Nasser, L.C.B. (Eds), Anais: biodiversidade e produção sustentável de alimentos e fibras nos cerrados: Brasília, 24 a 29 de março de 1996 / VIII Simpósio sobre o Cerrado, Planaltina, DF (Brasil), EMBRAPA-CPAC, 33-38.

Bravo, C., Florentino, A., 1999. Nivel de cobertura, conservación de suelos y aguas bajo diferentes sistemas de labranza, Revista Facultad de Agronomía, 25, 57-74.

${ }^{5}$ La mise en œuvre de telles démarches peut parfaitement s'intégrer dans les actions de réseaux comme le SMART (Strategic Monitoring of South-American Regional Transformations). 
Bravo, C., Hernández, R.M., Lozano, Z., Moreno, B., Piñango, L., 1999. Alternativas para el mejoramiento de la productividad del sistema maíz-ganado en suelos del Estado Guárico. Informe FONACIT proyecto S1-97001360, Venezuela.

Brossard, M., Lopes Assad, M.L., Chapuis, L., Barcellos, A. de O., 1997. Estoques de carbono em solos sob diferentes fitofisionomias de Cerrados, in Leite, L.L., Saito, C.H. (Eds), Contribuição ao conhecimento ecológico do Cerrado, [Brasília], Universidate de Brasília, Departamento de Ecologia, 272277.

Carvalho, R.B. de, Muniz, M., 1996. Árvore no chão ou enxofre no ar? O dilemma da siderurgia nas Minas Gerais, Ciência Hoje, 20, 38-47.

Chapuis-Lardy, L., Brossard, M., Quiquampoix, H., 2001. Assessing organic phosphorus status of Cerrado oxisols using 31P NMR spectroscopy and phosphomonoesterase activity measurement, Can. J. of Soil Science, 81, 591-601.

Dias, B.F. de S., 1993. Conservaçáo da natureza no cerrado brasileiro, in Novaes Pinto, M. (Ed.), Cerrado: caracterização e perspectivas, Brasília, Editora Universidade de Brasília, 608663.

Doran, J.W., Parkin, T.B., 1996. Quantitative indicators of soil quality: a minimum data set, in Doran, J.W., Jones, A.J. (Eds), Methods for Assessing Soil Quality, Madison (Wis.), Soil Science Society of America, 25-37.

Doran, J., Elliott, E.T., Paustian, K., 1998. Soil microbial activity, nitrogen cycling, and long-term changes in organic carbon pools as related to fallow tillage management, Soil Tillage Research, 49, 3-18.

Dumansky, J., 1993. Proceedings of the International Workshop on Sustainable Land Management for the 21st Century, Workshop Summary, Ottawa, Agricultural Institute of Canada.

FAO, 1992. Manual de sistemas de labranza para América Latina, Boletin de suelos de la FAO, Roma.

García-Guadilla, M.P., López-Hernández, D., 1998. Dilema de la productividad versus la sostenibilidad ecológica en el ecotono sabana-bosque del Amazonas venezolano, in Carrillo, R.J. (Ed.), Memorias del IV Congreso Interamericano sobre el Medio Ambiente, Caracas, Editorial Equinoccio, Ediciones de la Universidad Simón Bolívar, II, 282-286.

Goedert, W., 1983. Management of Cerrado soils of Brazil: A review, J. Soil Sci., 34, 405-423.

Henriques, R.P.B., 2003. O futuro ameaçado do Cerrado Brasileiro, Ciência Hoje, 33, 34-39.

Hernández, R.M., 1998. Transformaciones de carbono y nitrógeno y su distribución en agregados de un suelo tropical bajo dos tipos de labranza contrastante. Tesis Doctoral, Universidad Central de Venezuela, Caracas.

Hernández, R.M., Domínguez, C., 2002. Efecto de prácticas agrícolas usadas en distintas unidades de producción de maíz y sorgo sobre algunas propiedades bioquímicas de suelos del Estado Guárico, Agrobiológica, 2, 10-18.

Hernández, R.M., López-Hernández, D., 2002. Microbial biomass, mineral nitrogen and carbon content in savanna soil aggregates under conventional and no-tillage, Soil Biol. Biochem., 34, 1563-1570.

Hernández-Hernández, R.M., López-Hernández, D., 2002. El tipo de labranza como agente modificador de la materia orgánica: Un modelo para suelos de sabana de los Llanos centrales venezolanos, Interciencia, 27, 529-536.

Hernández-Valencia, I., López-Hernández, D., 1999. Allocation of phosphorus in a tropical savanna, Chemosphere, 39, 199207.
Hétier, J.-M., 2003. Factores limitantes de la fertilidad de los suelos llaneros, in Hétier, J.-M., López, R. (Eds), Tierras llaneras de Venezuela, Mérida (Venezuela), Centro Interamericano de Desarrollo e Investigación Ambiental y Territorial (CIDIAT), IRD, 293-334.

International Society of Soil Science (ISSS), 1998. Working Group R.B., World Reference Base for Soil Resources: Introduction, in Deckers, J.A., Nachtergaele, F.O., Spaargaren, O.C. (Eds), International Soil Reference and Information Centre (ISRIC) and Food and Agriculture Organization of the United Nations (FAO), Leuven (Belgique), Acco.

Jiménez, R.J., 1998. Efectos de diferentes sistemas de labranza sobre algunas propiedades físicas de un Fluventic Ustropept de los Llanos occidentales y el comportamiento del cultivo de maíz (Zea mays L.). Tesis Grado, UCV, Maracay.

Klink, C.A., 1996. Relação entre o desenvolvimento agrícola e a biodiversidade, in Pereira, R.C., Nasser, L.C.B. (Eds), Anais: biodiversidade e produção sustentável de alimentos e fibras nos cerrados: Brasília, 24 a 29 de março de 1996 / VIII Simpósio sobre o Cerrado, Planaltina, DF (Brasil), EMBRAPA-CPAC, 25-32.

Klink, C.A., Moreira, A.G., Solbrig, O.T., 1993. Ecological impact of agricultural development in the Brazilian Cerrados, in Young, M.D., Solbrig, O.T. (Eds), The world's savannas: Economic driving forces, ecological constraints and policy options for sustainable land use, Paris, Unesco, 259-282.

Kornelius, E., Saueressing, M.G., Goedert, W.J., 1979. Pasture establishment and management in the Cerrado of Brazil, in Sanchez, P.A., Tergas, L.E. (Eds), Pasture production in acid soils of the tropics, Cali (Colombia), CIAT, 147-166.

Lopes, A., Ayarza, M., Thomas, R., 1999. Sistemas Agropastoriles en las Sabanas de América Latina Tropical: Lecciones del Desarrollo Agrícola de los Cerrados de Brasil, in Guimarães, E.P., Sanz, J.I., Rao, I.M., Amézquita, M.C., Amézquita, E. (Eds), Publicación CIAT, 313, 9-30.

López-Hernández, D., 1995. Impact of agriculture and livestock production on tropical soils in Latin America, in Turner, B.L., Gómez-Sal, A., González Bernáldez, F., Castri, F. di (Eds), Global land use change: A perspective from the Columbian Encounter, Consejo Supremo de Investigaciones Cientificas (CSIC), 405-418.

López-Hernández, D., 1998. Agricultura de bajos insumos en el entorno sabana-bosque como un medio para reducir la presión sobre la selva tropical húmeda. Informe Final CONICIT Proyecto RP VII 290089.

López-Hernández, D., Ojeda, A., 1996. Alternativas en el manejo agroecológico de los suelos de las sabanas del norte de suramérica, Ecotropicos, 9, 99-115.

Lozano, Z., 1998. Evaluación de propiedades físicas y químicas de dos suelos de los Llanos occidentales con sistemas de labranza convencional y reducida. Tesis M.Sc., UCV, Maracay.

Marcano, F., Ohep, C., Francisco, D., 1993. Efectos de la labranza sobre algunas variables físicas en un suelo Oxic Haplustalf del Yaracuy medio bajo cultivo de maiz (Zea mays L.), Venesuelos, 1, 2-8.

MAS, 1994. Reunión del Steering Committee Management of Acid Soils, San José (Costa Rica), CATIE.

MAS, 1995. Reunión del Steering Committee Management of Acid Soils, Alemania, Feldafing.

Medina, E., 1987. Requirements, conservation and cycles of nutriments in the herbaceous layer, in Walker, B. (Ed.), Determinants of Tropical Savannas, Oxford, IRL Press, 36-65. 
Netuzhilin, Y., Chacón, P., Cerda, H., López-Hernández, D., Torres, F., Paoletti, M., 1999. Biodiversity tools to evaluate sustainability in savanna-forest Ecotone in the Amazon (Venezuela), in Reddy, M.V., Management of Tropical Agroecosystems and Benefical Soil Biota, Enfield (New Hampshire), Science Publishers Inc., 291-352.

Novais, R.F. de, 1996. A pesquisa em fertilidade do solo (no país) - Como a vejo (e a sinto), in Alvarez, V.V.H., Fontes, L.E.F., Fontes, M.P.F. (Eds), O solo nos grandes domínios morfoclimáticos do Brasil e o desenvolvimento sustendado, Viçosa (Brasil), Sociedade Brasileira de Ciência do Solo, Universidade Federal de Viçosa, Departamento de Solos, 397-409.

Oliveira, I.P., Kluthcouski, J., Yokoyama, L.P., Dutra, L.G., Portes, T.A, Silva, A. E., Pinheiro, B.S., Ferreira, E., Castro, E.M., Guimarães, C.M., Gomide, J.C., Balbino, L.C., 1996. Sistema Barreirão: Recuperação/renovação de pastagens degradadas em consórcio com culturas anuais, Goiânia (Brasil), EMBRAPA-CNPAF.

Oliveira, P.S., Marquis, R.J. (Eds), 2002. The cerrados of Brazil: ecology and natural history of a neotropical savanna, New York, Columbia University Press.

Pieri, C., 1989. Fertilité des terres de savanes : trente ans de recherche et de développement agricoles au sud du Sahara, Montpellier, CIRAD / Paris, Ministère de la Coopération et du Développement.

Pizarro, E.A., Valle, C.B. do, Keller-Grein, G., SchultzeKraft, R., Zimmer A.H., 1996. Regional experience with Brachiaria: Tropical America - Savannas, in Miles, J.W., Maass, B.L., Valle, C.B. do, Kumble, V. (Eds), Brachiaria: Biology, agronomy, and improvement, Cali (Colombia), Centro Internacional de Agricultura Tropical (CIAT) / Campo Grande (Brasil), Empresa Brasileira de Pesquisa Agropecuaria (EMBRAPA).

Pro-Cer/WWF, 1995. De grão em grão, o Cerrado perde espaço (cerrado - Impactos do processo de ocupação), Brasilia, WWF.

Resende, M., Ker, J.C., Bahia Filho, F.C., 1996. Desenvolvimento sustentado do cerrado, in Alvarez, V.V.H., Fontes, L.E.F., Fontes, M.P.F. (Eds), O solo nos grandes domínios morfoclimáticos do Brasil e o desenvolvimento sustendado, Viçosa (Brasil), Sociedade Brasileira de Ciência do Solo, Universidade Federal de Viçosa, Departamento de Solos, 169-199.

Rippstein, G., Lascano, C., Decaëns, T., 1996. La production fourragère dans les savanes d'Amérique du Sud intertropicale, Fourrages, 145, 33-52.

Rondón, M.A., Acevedo, D., Hernández, R.M., Pubiano, Y., Rivera, M., Amézquita, E., Romero, M., Sarmiento, L.,
Ayarza, M.A., Barrios, E., Rao, I., à paraître. Carbon sequestration of the neotropical savanna (Llanos) of Colombia and Venezuela, in Rattan Lal, R., Kimble, J. (Eds), Potential of soil carbon sequestration in Latin America, CRP Press (Pays-Bas).

Sá, J.C., 1999. Manejo da fertilidade do solo no sistema plantio direto, in Siqueira, J.O., Moreira F.M.S., Lopes, A.S., Guilherme, L.R.G., Faquin, V., Furtini, A.E., Carvalho, J.G. (Eds), Inter-relação fertilidade, biologia do solo e nutrição de plantas, Lavras (Brasil), SBCS/Universidade Federal de Lavras, 267-320.

Sánchez, C., 1989. Contribución al conocimiento de la mínima labranza en Venezuela, San Juan de los Morros (Venezuela), Universidad Nacional Experimental de los Llanos Centrales Rómulo Gallegos.

Scopel, E., Douzet, J.M., Macena da Silva, F.A., Cardoso, A., Alves Moreira, J.A., Findeling, A., Bernoux, M., 2005. Impact des systèmes de culture en semis direct avec couverture végétale (SCV) sur la dynamique de l'eau, de l'azote minéral et du carbone du sol dans les cerrados brésiliens, Cahiers Agricultures, 14, 1, 71-75.

Seguy, L., Bouzinac, S., Trentini, A., Côrtes, N.A., 1996. L'agriculture brésilienne des fronts pionniers, Agriculture et développement, 12, 2-61.

Siqueira, J.O., Moreira, F.M.S., 1999. Inter-relação fertilidade, biologia do solo e nutrição mineral de plantas: base para um novo paradigma na agrotecnologia do século XXI, in Siqueira, J.O., Moreira, F.M.S., Lopes, A.S., Guilherme, L.R.G., Faquin, V., Furtini, A.E., Carvalho, J.G. (Eds), Interrelação fertilidade, biologia do solo e nutrição de plantas, Lavras (Brasil), SBCS/Universidade Federal de Lavras, 1-10.

Spain, M.J., Ayarza, M.A., Vilela, L., 1996. Crop pasture rotations in the brazilian Cerrados, in Pereira, R.C., Nasser, L.C.B. (Eds), Anais: biodiversidade e produção sustentável de alimentos e fibras nos cerrados: Brasília, 24 a 29 de março de 1996 / VIII Simpósio sobre o Cerrado, Planaltina, DF (Brasil), EMBRAPA-CPAC, 39-45.

Stocking, M.A., 2003. Tropical soils and food security: the next 50 years, Science, 302, 1356-1359.

Théry, H., Mello, N.A. de, 2005. Diversités et mobilités de l'agriculture brésilienne, Cahiers Agricultures, 14, 1, 11-18.

Van Wambeke, A., 1971. Recherches sur la mise en valeur agricole des sols acides des savanes arborées du Brésil, Pédologie, 21, 2, 211-255.

Waniez, P., 1992. Les CERRADOS, “ un espace frontière " brésilien, Montpellier, RECLUS / Paris, ORSTOM.

Reçu le 7 juillet 2004. Accepté le 12 mai 2005. 